relationship between otherwise distant transmembrane and ATPase modules.

\section{F0924線虫イネキシン 6 ギャップ結合チャネルの単離と機能特性}

Isolation and characterization of $\mathrm{C}$. elegans innexin- 6 gap junction channels

Atsunori Oshima ${ }^{1}$, Tomohiro Matsuzawa ${ }^{2}$, Kouki Nishikawa', Yoshinori Fujiyoshi ${ }^{1}\left({ }^{1}\right.$ CeSPI, Nagoya Univ., ${ }^{2}$ Grad. Sch. of Sci., Kyoto Univ.)

Invertebrates possess gap junction channels composed of innexins that evolved independently from vertebrate connexins. Only limited information is, however, available on the innexin channel structure. Here we successfully expressed and purified $C$. elegans innexin-6 (INX-6, 45kDa) gap junction channels, and characterized the structural properties and channel permeability using electron microscopy and microinjection of fluorescent dye tracers, respectively. The INX-6 proteins formed gap junction plaques with a loosely packed hexagona lattice. Channel distance, based on the reflection spots of the INX-6 gap junction plaques, was longer than that of connexin-26 (Cx26, $26 \mathrm{kDa})$. Thin-section electron microscopy revealed that the INX-6 junction was thicker than the Cx26 junction. The purified INX-6 channels in dodecylmaltoside solution generally ran slower than Cx26 channels in gel filtration analysis, suggesting that the purified INX- 6 channels were mostly hemichannels, which was supported by electron microscopy and native gel electrophoresis. Dye transfer experiments showed that 3-kDa dextran-Texas red and 10-kDa dextran-Texas red passed through INX-6 channels at rates of approximately $50 \%$ and $10 \%$, respectively, while no significant amounts passed through connexin- 43 channels. These results have implications regarding the dimensions and overall structure of the INX-6 channels, and provide insight into the wider pore diameter of INX-6 channels compared with that of connexin channels.

\section{F0936 ATR-FTIR 法を用いた KesA 野生型と不活化しない变異体に文} するカチオン誘起棰造变化の赤外分光分析

Cation-induced structural changes in the WT and noninactivating mutant KesA studied by ATR-FTIR

Chikako Muramatsu ${ }^{1}$, Masayo Iwaki ${ }^{1}$, Tetsuya Fukuda', Yusuke Asai ${ }^{1}$, Yuji Furutani ${ }^{1,2}$, Hideki Kandori ${ }^{1}$ ( ${ }^{1}$ Grad. Sch. Tech., Nagoya Inst. Tech., ${ }^{2}$ Inst. Mol. Sci.,)

The bacterial potassium channel KcsA is a membrane protein complex of homotetramer. The channel is gated by high concentration of intracellular protons, and transports cations through the filter that permeates $\mathrm{K}^{+}$selectively over $\mathrm{Na}^{+}$. In the wild type (WT) KcsA, the filter can be in both open and inactivated (collapsed) structures depending on ionic/pH conditions, whereas the filter remains open in the non-inactivating mutants such as E71A, E71V and F103A. In this study, we investigate the mechanism of ion selectivity coupled with gating process using attenuated total reflection Fourier transform infrared (ATR-FTIR) spectroscopy. WT and mutant proteins of KcsA of Streptmyces lividans were expressed in $E$. coli, and purified proteins were reconstituted in liposomes and deposited on an ATR-prism. $\mathrm{K}^{+}$- or $\mathrm{Na}^{+}$-induced difference FTIR spectra were measured by switching perfusion buffers with and without cation. At pH 7, the gate of the WT was closed and the selectivity filter was presumably open. $\mathrm{K}^{+}$-induced spectrum showed different IR features from $\mathrm{Na}^{+}$induced one, indicating different ionic interactions of $\mathrm{K}^{+}$and $\mathrm{Na}^{+}$to the protein. The IR spectra obtained at $\mathrm{pH} 4$ were different from those at $\mathrm{pH} 7$. The corresponding spectra measured in the non-inactivating mutants were compared to those in the WT. Conformational changes in peptides upon the binding of cations to KcsA are discussed based on the obtained IR data in the light of atomic structures available from X-ray crystallography.

\section{F0948 黄色ブドウ球菌の 2 成分性膜孔形成害素の分子譏構}

Molecular basis of staphylococcal bi-component pore forming toxin

Yoshikazu Tanaka ${ }^{1}$, Daichi Yamashita ${ }^{2}$, Keitaro Yamashita $^{2}$, Yuka Kawai $^{2}$, Jun Kaneko ${ }^{3}$, Noriko Tomita ${ }^{4}$, Makoto Ohta ${ }^{4}$, Yoshiyuki Kamio ${ }^{5}$, Min Yao', Isao Tanaka ${ }^{1}{ }^{1}$ Fac. of Adv. Life Sci., Hokkaido Univ., ${ }^{2}$ Grad. Schl. of Life Sci., Hokkaido Univ., ${ }^{3}$ Grad. Schl. of Agri. Sci., Tohoku Univ., Inst. of Fluid Sci., Tohoku Univ., ${ }^{5}$ Grad. Schl. of Life Sci., Tohoku Univ.)

Pathogenic bacteria secrete various pore-forming toxins (PFTs) to kill host cells. PFTs are expressed as water-soluble monomeric proteins that oligomerize into nonlytic prepore intermediates on the membrane of the target cells, and then form bilayer-spanning pores.

Staphylococcus aureus, a ubiquitous and pernicious human pathogen, secretes several PFTs including $\alpha$-hemolysin $(\alpha \mathrm{HL}), \gamma$-hemolysin $(\gamma \mathrm{HL})$, leukocidin (LUK), and Panton-Valentine leukocidin (PVL). $\alpha \mathrm{HL}$ consists of a single polypeptide, whereas the others are bi-component PFTs that require the synergistic association of a class $\mathrm{F}$ component and a class $\mathrm{S}$ component. Extensive experiments have been carried out for more than two decades, and the crystal structures of the monomeric forms of bi-component PFTs have been determined. However, the structures of the prepore and pore forms have not been reported at atomic resolution, which has hindered detailed discussion of the complicated molecular mechanism.

In the present study, we determined the crystal structure of the prepore and pore form of bi-component PFT, $\gamma \mathrm{HL}$. This is the first report of the crystal structure of a heterocomponent $\beta$-barrel-type transmembrane protein. This is also the first PFT of which all of monomer-, prepore-, and pore-form structures have been determined by X-ray crystallography. Based on the structural differences between pore and monomer forms in combination with biological data accumulated over the past two decades, we propose a mechanism of pore formation by PFTs.

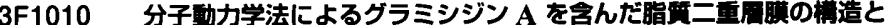 圧力特性}

Structure and lateral pressure profile of lipid bilayer containing gramicidin A by molecular dynamics simulation

Hiroaki Saito, Masashi Iwayama, Megumi Nishimura, Hiroyuki Takagi, Kazutomo Kawaguchi, Hidemi Nagao (Kanazawa University)

The molecular dynamics (MD) simulations of DMPC (diC14:0-PC) and DSPC (di18:0-PC) lipid bilayers in the absence and presence of gramicidin A (GA) peptide dimer were carried out to investigate the GA effect on these membrane structure and the lateral structure. The observed hydrophobic thickness and order parameter increased, and the gauche conformation reduced in the presence of GA, and these results are consistent with the experimental measurements. Due to the hydrophobic mismatch between the GA and surrounding lipid bilayer, the hydrophobic thickness of the DSPC membrane largely decreased to fit the length of hydrophobic core region of the GA peptide dimer, resulting in a distortion of the membrane surface around the GA. The surface tension around the polar headgroup region decreased due to the decrease of electrostatic interaction between headgroups. Since the DSPC lipid bilayer has a larger hydrophobic length, the larger positive lateral pressure appears around the GA channel entrance region in the membrane. The larger lateral pressure in the hydrophobic region of the DSPC/GA membrane results in the reduction of pore radius around the GA channel entrance, suggesting the decrease of ion accessibility to the GA channel entrance.

\section{F1022 代謝型グルタミン酸受容体の膜具通領域における 1 アミノ酸変異が アゴニストをインハーースアゴニストに変える}

Single amino acid substitution in the transmembrane domain of metabotropic glutamate receptor changes an agonist into an inverse agonist

Masataka Yanagawa, Takahiro Yamashita, Yoshinori Shichida (Department of Biophysics, Graduate School of Science, Kyoto University)

Metabotropic glutamate receptors (mGluRs) are prototypical family $3 \mathrm{G}$ protein-coupled receptors (GPCRs) that function as a constitutive homo dimer. They have a large extracellular ligand-binding domain (ECD) on the N-terminal side of seven-transmembrane domain (TMD). Upon binding of an agonist, an intersubunit relocation of the dimeric ECDs induces a mutual rearrangement of TMDs, which was observed by our previous fluorescence resonance energy transfer (FRET) analyses. However, the conformational change within each protomer of mGluR is largely unknown. Here we report that a single amino acid mutation at a threonine residue conserved in helix VI of mGluRs caused elevation of a basal activity, whereas the glutamate binding to the mutant partially suppressed the basal activity. Namely, original "agonist" works as an "inverse agonist" on this mutant. The comparative FRET analysis of wild-type and the mutant demonstrated that the glutamate-dependent dimeric rearrangement of the mutant is similar to that of wild-type. Therefore, alteration of the ligand character in the mutant should be originated from a conformational change within each protomer. Double mutation analyses revealed that an additional mutation in helix VII restored the "agonist"-dependent increase of the activity. In conclusion, the proper packing between helices VI and VII around the threonine residue within each protomer is a key determinant of the activation of mGluRs. 